\title{
Thermal wave and thermal pulse studies of the impact of manufacturing technology on the polarization of piezofiber composites
}

\author{
A. Eydam ${ }^{1}$, G. Suchaneck ${ }^{1}$, G.Gerlach ${ }^{1}$ \\ ${ }^{1}$ Technische Universität Dresden, Solid State Electronics Laboratory, 01062 Dresden, Germany \\ agnes.eydam@tu-dresden.de
}

\begin{abstract}
In this work, we apply the thermal pulse method to study the impact of process steps on the polarization of a piezofiber composite being integrated in a thermoplastic structure. Pre-poled samples are compared with samples which are poled after each integration step. The thermal wave method investigated previously shows a significant difference between these sample groups. The pyroelectric current is decreased by the embedding into thermoplastics. Contrarily, the differences between prepoling and re-poling are less verifiable when using the thermal pulse method.
\end{abstract}

Key words: thermal pulse method, laser intensity modulation method, polarization, piezofiber composite, integration.

\section{Introduction}

Smart active systems based on integrated piezoceramics find applications for active noise control, condition monitoring or structural health monitoring of safety components [1]. In practice, their polarization state is affected by mechanical and thermal loads appearing during device fabrication. Thermal wave and thermal pulse methods are non-destructive ways to study the influence of process steps on the ferroelectric polarization.

In the Laser Intensity Modulation Method (LIMM), the piezoelectric transducer is heated by an intensity-modulated laser beam. The pyroelectric current is recorded in frequency domain, whereas a time domain signal is measured in the thermal pulse method after heating with laser pulses. The signal of latter can be Fourier-transformed and analyzed in frequency domain similar to LIMM. Recently, we have demonstrated that the real part of the pyroelectric current spectra obtained from thermal pulse measurements by Fourier transform is in good agreement with the LIMM spectra when the influence of the transfer function of the measurement setup is corrected [2]. The use of thermal pulses instead of thermal waves enables a significantly shorter measuring time [3].

In this work, we apply both methods to investigate the polarization state of piezofiber composites during the integration steps leading to an active fiber-reinforced plastic structure.

\section{Experimental}

Piezofiber composites (PFC) consisting of a monolayer of PZT fibers embedded in epoxy resin were used as starting material [4]. In the first integration step, they were embedded between two transparent thermoplastic carrier films of Polyamide 6 and consolidated by hotpressing, forming the thermoplastic-compatible piezoceramic module (TPM). In the second integration step, these TPMs were integrated into a fiber-reinforced plastic (FRP) structure [5]. The investigated samples were divided into two groups [6]:

- pre-poled samples, which were poled as PFCs and integrated,

- re-poled samples, which were additionally poled after each integration step.

LIMM measurements were performed by heating the samples periodically by a single laser diode (LCU98A041A, Laser Components $\mathrm{GmbH}$, Olching, Germany) square-wavemodulated in the frequency range from $100 \mathrm{mHz}$ up to $1 \mathrm{kHz}$ with a power of $12 \mathrm{~mW}$ at a wavelength of $980 \mathrm{~nm}$. The complex pyroelectric current was determined by an impedance/gain-phase analyzer (Solartron 1260, Solartron Analytical, Farnborough, UK) 
with DC coupling. In order to reduce noise, 30 measurement repetitions were used for averaging.

Thermal pulse measurements were carried out by heating the samples with a pulsed laser diode (LC905D3S3J09S, Laser Components $\mathrm{GmbH}$, Olching, Germany) at a wavelength of $905 \mathrm{~nm}$ with a peak power of $55 \mathrm{~W}$, a pulse width of $100 \mathrm{~ns}$ and a repetition frequency of $1 \mathrm{~Hz}$. The pyroelectric current was transformed to a voltage by a current amplifier (SR570, Stanford Research Systems, Sunnyvale, CA) and filtered by a home-built $50 \mathrm{~Hz}$ notch filter before recording by a Waverunner ${ }^{\circledR}$ Xi-A oscilloscope (LeCroy, Chestnut Ridge, USA) with DC coupling.

\section{Theory}

The pyroelectric current is described by a fundamental relation [7]:

$$
I(t)=\frac{A}{d} \int_{0}^{d} p(z) \frac{\partial}{\partial t} \Theta(z, t) d z,
$$

where $A$ is the heated area, $d$ the piezoelectric film thickness, $p(z)$ the pyroelectric coefficient distribution and $\Theta=T-T_{0}$ the temperature difference to the environment.

Assuming a homogeneous polarization equivalent to $p(z)=p_{0}=$ const., eq. (1) simplifies to:

$$
I(t)=A p_{0} \frac{d \Theta_{m}}{d t},
$$

where $\Theta_{m}(t)$ is the temperature averaged across the sample.

In the case of LIMM, the pyroelectric current of a homogeneously poled piezoceramic exhibiting thermal losses to the environment yields [8]:

$$
I(\omega, t)=\frac{\Phi_{0} A}{c \rho \cdot d} p_{0} \frac{i \omega \tau_{t h}}{1+i \omega \tau_{t h}} \exp (i \omega t),
$$

with the heat flux $\Phi_{0}$ absorbed by the plate surface, the specific heat $c$, the density $\rho$ and the thermal relaxation time $\tau_{\text {th }}$.

The thermal relaxation time of $\mathrm{PZT}$ fibers embedded in epoxy resin exceeds a few seconds. Thus, the thermal losses occur in a frequency range below $0.1 \mathrm{~Hz}$. Also, the piezoelectric material causes piezoelectric resonances in the $\mathrm{kHz}-$ and $\mathrm{MHz}$-range [9]. Thus, eq. (3) simplifies in the frequency region from $0.1 \mathrm{~Hz}$ to $100 \mathrm{~Hz}$ to:
$I(\omega)=\frac{\Phi_{0} A}{c \rho \cdot d} p_{0} \exp (i \omega t)$.

In the case of the thermal pulse method, the pyroelectric response can be described in the time domain with eq. (2) if the temperature distribution $\Theta(z, t)$ in the piezoelectric material is known $[10,11]$ or it can be converted to the frequency domain by a Fourier transform. The obtained frequency spectra are corrected by the transfer function of the measurement set-up to account for the influence of the amplifier settings. This approach was described in detail in [2].

According to eqs. (2) and (4), the ratio of the pyroelectric currents of two samples will be equal to the ratio of their pyroelectric coefficients when the absorbed laser radiation and the temperature distribution are also equal. This holds in our case since the laser beam is always absorbed at the gold electrode of the PFC and heat losses are negligible.

\section{Results and Discussion}

The influence of the integration process on the polarization of the PFC was investigated by LIMM in [6]. The pyroelectric current and correspondingly the remanent polarization of the pre-poled samples decrease after integration into the thermoplastic structure. In contrast, the polarization of the re-poled samples increases or decreases only slightly.

In this work, the same samples were studied by the thermal pulse method. Fig. 1 shows the pyroelectric current spectra of a pre-poled and a re-poled sample in the three states PFC, TPM and FRP, obtained by LIMM and by the thermal pulse method, respectively. The LIMM spectra were almost constant in the frequency range between 1 and $10 \mathrm{~Hz}$. At low frequencies, thermal losses affect the current and at high frequencies piezoelectric resonances are visible. The pyroelectric response in time domain was measured at different bandwidths of the current amplifier. A bandwidth of $200 \mathrm{~Hz}$ was chosen in fig. 1 because the spectra at a lower bandwidth of $15 \mathrm{~Hz}$ were attenuated at frequencies below $10 \mathrm{~Hz}$ due to the influence of AC coupling. A decrease of the pyroelectric current was obtained for both samples. The difference made by re-poling is difficult to verify. There are several issues which affect the spectra obtained by the thermal pulse method. First, they are noisier than the LIMM spectra because the heat pulse is distributed over the whole frequency spectrum. Second, using LIMM, each frequency point was measured separately with 30 repetitions. Applying the 
thermal pulse method, the time domain signal was averaged only over 10 measurements to balance it with the increasing acquisition time. Third, there are the already mentioned effects at low and high frequencies.

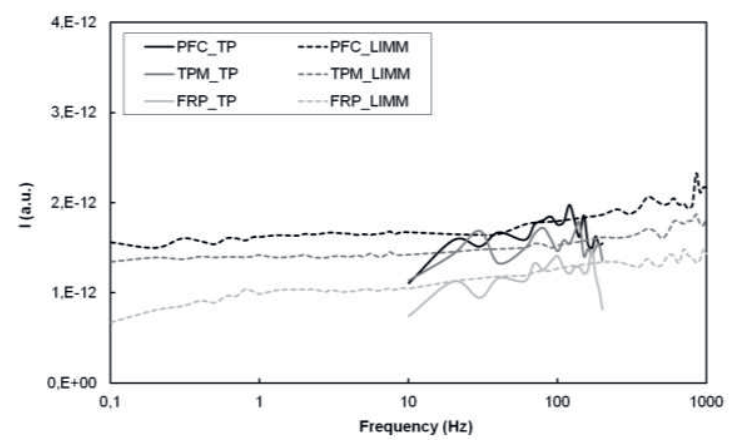

(a)

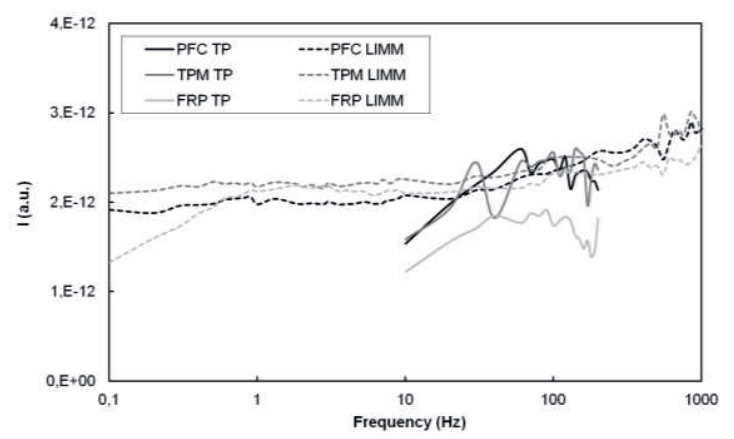

(b)

Fig. 1. Comparison of the real parts of the pyroelectric current spectra of (a) a pre-poled sample and (b) a re-poled sample in the three states PFC, TPM and FRP obtained by the thermal pulse method (TP) for a bandwidth of $200 \mathrm{~Hz}$ and by LIMM.

The corresponding signals of the pyroelectric response in time domain are shown in fig. 2 . Here, the difference between pre-poling and repoling is observable if PFC and TPM are compared. For the FRP, the current decreases in both cases. The ratio of the averaged currents of the spectra and the ratio of the peak currents both at $200 \mathrm{~Hz}$ bandwidth are equal if two states of the same sample are compared. But these ratios differ from the ones of the LIMM spectra.

There is still potential for improvement of the thermal pulse method by further noise reduction but the achievable accuracy of the measurement method seems to be limited.

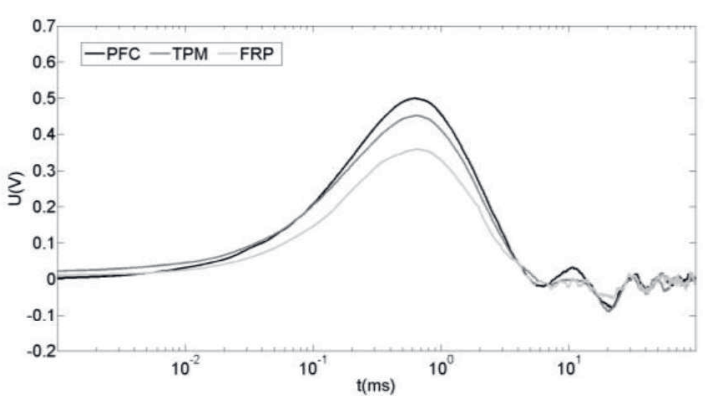

(a)

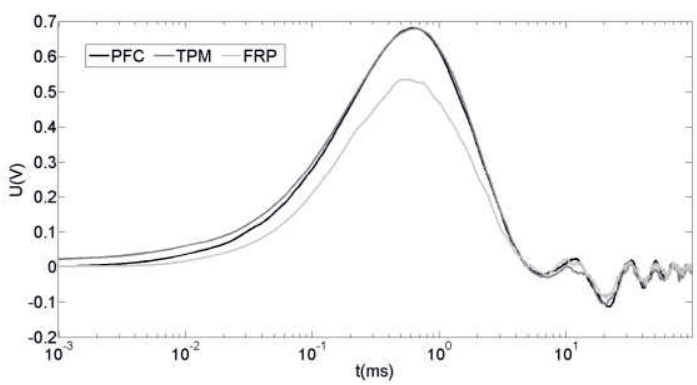

(b)

Fig. 2. Thermal-pulse response at a bandwidth of $200 \mathrm{~Hz}$ of (a) a pre-poled sample and (b) a re-poled sample in the three states PFC, TPM and FRP.

\section{Conclusions}

The manufacturing technology affects the polarization state of piezofiber composites. LIMM experiments revealed a degradation of the pyroelectric current after integration in thermoplastic components when the samples were not re-poled. The thermal pulse method is still not accurate enough to verify the difference between pre-poled and re-poled samples.

\section{Acknowledgement}

This research is supported by the Deutsche Forschungsgemeinschaft (DFG) in context of the Collaborative Research Centre/Transregio 39 PT-PIESA, subproject C8.

\section{References}

[1] J. Qiu, H. Ji, The application of piezoelectric materials in smart structures in China, International Journal of Aeronautical and Space Sciences 11, 266-284 (2010)

[2] A. Eydam, G. Suchaneck, G. Gerlach, Thermal pulse method for non-destructive characterization of integrated piezoelectric transducers, In: Eurosensors XXX, 2016, Procedia Engineering $168,848-851(2016)$ 
[3] A. Mellinger, R. Singh, R. Gerhard-Multhaupt, Fast thermal-pulse measurements of spacecharge distributions in electret polymers, Review of Scientific Instruments 76, 013903 (2005)

[4] K. Hohlfeld, P. Neumeister, S. Gebhardt, A. Michaelis, Tailored Composite Transducers Based on Piezoceramic Fibres and Pearls, Proceedings ACTUATOR 16, Bremen, Germany, June 13-15, 2016, 69-72 (2016)

[5] W. Hufenbach, M. Gude, N. Modler, Th. Heber, A. Winkler, T. Weber, Process chain modeling and analysis for the high-volume production of thermoplastic composites with embedded piezoceramic modules, Smart Materials Research 2013, 201631 (2013)

[6] K. Hohlfeld, S. Eßlinger, A. Eydam, A. Winkler, T. Weber, M. Gude, N. Modler, G. Gerlach, G. Suchaneck, A. Michaelis, A. Schönecker, S. Gebhardt and P. Neumeister, Effect of the integration of piezoceramic composites into structural components on their poling condition and polarizability, 8th ECCOMAS Thematic Conference on Smart Structures and Materials (SMART 2017), June 5 - 8, 2017, Madrid, Spain (accepted for publication )

[7] B. Ploss, R. Emmerich, S. Bauer, Thermal Wave Probing of Pyroelectric Distributions in the Surface Region of Ferroelectric Materials - a New Method for the Analysis, Journal of Applied Physics 72, 5363-5370 (1992)

[8] S. Bauer, B. Ploss, A method for the measurement of the thermal, dielectric, and pyroelectric properties of thin films and their applications for integrated heat sensors, Journal of Applied Physics 68, 6361-6367 (1990)

[9] S. Bauer, S. Bauer-Gogonea, Current Practice in Space Charge and Polarization Profile Measurements Using Thermal Techniques, IEEE IEEE Transactions on Dielectrics and Electrical Insulation 10, No. 5, 883-902 (2003)

[10] A. Eydam, G. Suchaneck, G. Gerlach, Characterization of the Polarization State of Embedded Piezoelectric Transducers by Thermal Waves and Thermal Pulses, Journal of Sensors and Sensor Systems, 5, 165-170 (2016)

[11] A. Eydam, G. Suchaneck, G. Gerlach, Thermal excitation as a mean for nondestructive evaluation of embedded piezoelectric transducers, IEEE Conference Proceedings on ISAF/ECAPD/PFM, August 21-25, 2016, Darmstadt (Germany) 